\title{
The Application of Molecularly Imprinted Polymers
}

\author{
Ming Zhao, Guijun Shen* \\ College of Environmental and Chemical Engineering, Dalian University, Dalian, China \\ Email: sgj0501@163.com
}

Received 16 April 2015; accepted 24 June 2015; published 1 July 2015

\begin{abstract}
Molecularly imprinted technology (MIT) has the characteristics of specificity and high selectivity, which is one of the most promising methodologies. Besides, the polymers are made using MIT as the functional material of solid-phase extraction and chromatographic fractionating and sensor, because of the characteristics of the high selectivity, the better stability and easy preparation. This review introduces the progress in the application of MIT and summarizes its application in the chemistry.
\end{abstract}

Keywords

Molecularly Imprinted Polymers, Solid Phase Extraction, Chromatographic Fractionating

\section{Introduction}

Molecular imprinting received a wide range of concerns since the publicly synthesis of molecularly imprinted polymers by Wulff in 1972 [1]. Molecular imprinting technology originated in antigen as template to synthesize antibodies that the template molecule, specific functional monomers and cross-linking agents are to obtain solid polymers. Molecularly imprinted polymers (MIPs), as man-made material of antibodies, possess recognition sites within the polymers that are adapted to the three-dimensional shape [2].

\section{Preparation of MIPs}

Combin template and functional monomer in the specific dispersion system, adding cross-linking agent (or adding sorbent) and initiator, and then that will get the high alternate and robust polymer by non-covalent interaction or reversible covalent interaction [3] [4]. Consequentially, the template molecule was washed out from the polymer under harsh physics or chemistry conditions, which obtain a three-dimensional hole can identify the template molecule [5]. MIPs are gaining in importance as robust molecular recognition elements in analytical and separation sciences. What we can do is just to overcome the difficulty of choosing the appropriate conditions by chemical synthesis.

According to the property of the additive polymer made by template and functional monomers, there are covalent imprinting and non-covalent imprinting. Covalent imprinting, in which the process is explicit, distinct,

${ }^{*}$ Corresponding author. 
steady and hard, is the less used technology for preparing MIPs than non-covalent imprinting, in which the complex of template and functional monomer is formed in situ by non-covalent interactions. Non-covalent interactions is the widely used, because the interaction is relative quick, and the template molecule was washed out from the polymer in the temperate condition. What is more, a planty of the functional monomers will have non-typical binding point, so that it deduces the ability of the selectivity of the synthetic substrate. There are several polymerization method such as suspension polymerization, situ polymerization, bulk polymerization, surface polymerization, dispersion polymerization, precipitation polymerization and swelling polymerization. Above all, bulk polymerization is the most common method in which template, functional monomer, crosslinking agent, adding sorbent and initiator dissolve in an inert solvent in appropriate proportions, which carry out in the light or thermal initiation under anaerobic conditions. We will get the particle what size we want through polymers obtained by crushing, grinding and sieving.

\section{The Application of MIPs}

Based on the relevant characteristics of MIPs compared to other adsorbents such as selectivity, stability, sensitivity, high lifetime, etc. we perceive, it deserve arousing widely concern. The technique have already brought forward, successfully applying to solid phase extraction, chromatographic fractionating and bionic sensor.

\subsection{Applications to Solid Phase Extraction (SPE)}

Compared to the traditional extradition sorbent, MIPs are much better matched to the template molecule, so that they can identify the extract specifically. Particularly, MIPs, as the solid phase extraction, can reduce the question about complicated environmental sample system factor and heavy pretreatment factor, and then MIPs is widely used as solid phase material to separate and enrich (Table 1).

\subsection{Applications to Chromatographic Fractionating}

Chromatographic methods, including HPLC, HPLC-MS and GC-MS [10], are able to provide molecular structure identification of the captured molecules. In order to reduce the limits of detecting instrument, we attach more importance to the effective enrichment approach. Obviously, MIPs is suited to be the analytical column and to separate sample (Table 2).

\subsection{Applications to Sensor}

Recently, molecularly imprinted polymers (MIPs), as a recognition element for sensors, have made a great progress. Chemical (or biological) sensor consists of a recognition material and a response signal component. In

Table 1. Application of MIPs to SPE.

\begin{tabular}{|c|c|c|c|c|}
\hline Template & Function monomer & Polymerization & Sample & Reference \\
\hline Iphenylguanidine & Iphenylguanidine & Bulk polymerization & Water & {$[6]$} \\
\hline Lidocaine & Itaconic acid & Bulk polymerization & Serum & [7] \\
\hline Methyltestosterone & Methacrylic acid & Bulk polymerization & Hormone & {$[8]$} \\
\hline Dimethomorph & Methacrylate & $\begin{array}{l}\text { Bulk polymerization and } \\
\text { suspension polymerization }\end{array}$ & Antiseptic & [9] \\
\hline
\end{tabular}

Table 2. Application of MIPs to chromatographic fractionating.

\begin{tabular}{cccc}
\hline Template & Function monomer & Sample & Analytical system \\
\hline Ractopamine & Acrylamide & Feed & MISPE-HPLC-FLD \\
Triadimenol & Methacrylate acid & Food & MISPE-HPLC \\
Nicotinamide & MAA & Drug & MISPE-HPLC-UV \\
\hline
\end{tabular}


general, MIP film acts as the signal transducer that converts the binding signals from the molecular recognition to detectable electrical signals, and has an advantage of sensitivity of the sensing system [14].

\section{References}

[1] Feng, Y.Q., Zhou, R.J., Tang, Y.B. and Hu, X.X. (2011) The Application of Molecularly Imprinted in Solid Phase Extraction. PTCA (PARTB: CHEM, ANAL), 47, 125-128.

[2] Yan, H.Y., Wang, F. and Han, D.D. (2012) Simultaneous Determination of Four Plant Hormones in Bananas by Molecularly Imprinted Solid-Phase Extraction Coupled with High Performance Liquid Chromatography. ANALYST, 137, 2884-2890. http://dx.doi.org/10.1039/c2an35362h

[3] Djozan, D. and Ebrahimi, B. (2008) Preparation of New Solid Phase Micro Extraction Fiber on the Basis of AtrazineMolecular Imprinted Polymer: Application for GC and GC/MS Screening of Triazine Herbicides in Water, Rice and Onion. Analytical Chemical Acta, 616, 152-159. http://dx.doi.org/10.1016/j.aca.2008.04.037

[4] Caro, E., Marcel, R.M., Cormack, P.A.G., Sherrington, D.C. and Borrull, F. (2003) On-Line Solid-Phase Extraction with Molecularly Imprinted Polymers to Selectively Extract Substituted 4-Chlorophenols and 4-Nitrophenol from Water. Chromatogram A, 995, 233-238. http://dx.doi.org/10.1016/S0021-9673(03)00543-0

[5] Wulff, G. (1995) Molecular Imprinting in Cross-Linking Materials with the Aid of Molecular Templates a Way towards Artificial Antibodies. Angewandte Chemie International Edition, 34, 1812-1832. http://dx.doi.org/10.1002/anie.199518121

[6] Wei, Y.F., Fan, J. and Shao, X.J. (2008) Preparation of Diphenylguanidine-Imprinted Polymer and Its Application for Solid-Phase Extraction. Journal of Instrumental Analysis, 10, 1039-1043.

[7] Hu, Y.F., Zhang, Z.H., Zhang, H.B., Luo, L.J. and Yao, S.Z. (2012) Selective and Sensitive Molecularly Imprinted SolGel Film-Based Electrochemical Sensor Combining Mercaptoacetic Acid-Modified PbS Nanoparticles with $\mathrm{Fe}_{3} \mathrm{O}_{4} @ \mathrm{Au}-$ Multi-Walled Carbon Nanotubes-Chitosan. Journal of Solid State Electrochemistry, 16, 857-867. http://dx.doi.org/10.1007/s10008-011-1434-4

[8] Yng, M.L., Shen, R.L., Gu, W.C., Chu, X.G. and Wang, D.N. (2012) Study on Methyltestosterone Imprinted Polymers and Its Application in Solid Phase Extraction. Chinese Journal of Veterinary Drug, 12, 6-8.

[9] Wng, Y., Xu, X.W. and Ling, S. (2010) Synthesis of Dimethomorph Imprinted Polymers and Molecularly Imprinted Solid-Phase Extraction Analysis. Guangdong Journal of Chemistry, 37, 7.

[10] Shi, Y., Peng, D.D., Shi, C.H., Zhang, X. and Xie, Y.T. (2011) Selective Determination of Trace 17 $\beta$-Estradiol in Dairy and Meat Samples by Molecularly Imprinted Solid-Phase Extraction and HPLC. Food Chemistry, 126, 19161925. http://dx.doi.org/10.1016/j.foodchem.2010.12.020

[11] Huang, Y., Zhang, Q.J. and Liu, M. (2012) Molecularly-Imprinted Solid Phase Extraction Coupled with High Performance Liquid Chromatography for the Determination of Ractopamine in Feed Samples. Chinese Journal of Chromatography, 1, 56-61.

[12] Hu, J., Wu, X.Y. and Gao, W.H. (2012) HPLC Analysis of Two Triazole Fungicide Residues by Molecularly Imprinted Solid Phase Extraction. Chinese Journal of Pharmaceutical Analysis, 32, 6.

[13] Li, Z.W., Liu, S.B. and Yang, G.L. (2005) Fast Seperation of Nicotinamide and Nicotinic Acid with Molecularly Imprinted Monolithic Column. Chinese Journal of Chromatography, 6, 622-655.

[14] Liu, C., Muhammad, T., Piletska, E.V. and Zeng, H.-H. (2010) Synthesis of Lidocaine Molecularly Imprinted Polymers and Application on Solid Phase Extraction. Chinese Journal of Analytical Chemistry, 11, 1652-1656. 